\title{
Arrêts sur vingt-sept images de l'Europe
}

\section{Christophe Didier}

\section{(2) OpenEdition}

Journals

Édition électronique

URL : http://journals.openedition.org/rbnu/640

DOI : 10.4000/rbnu.640

ISSN : 2679-6104

\section{Éditeur}

Bibliothèque nationale et universitaire de Strasbourg

\section{Édition imprimée}

Date de publication : 1 novembre 2017

Pagination : 6-7

ISBN : 9782859230678

ISSN : 2109-2761

\section{Référence électronique}

Christophe Didier, "Arrêts sur vingt-sept images de l'Europe », La Revue de la BNU [En ligne], 16 | 2017, mis en ligne le 01 juillet 2019, consulté le 19 décembre 2020. URL : http://journals.openedition.org/ rbnu/640; DOI : https://doi.org/10.4000/rbnu.640

\section{(C) $10(0$}

La Revue de la BNU est mise à disposition selon les termes de la Licence Creative Commons Attribution - Pas d'Utilisation Commerciale - Partage dans les Mêmes Conditions 4.0 International. 


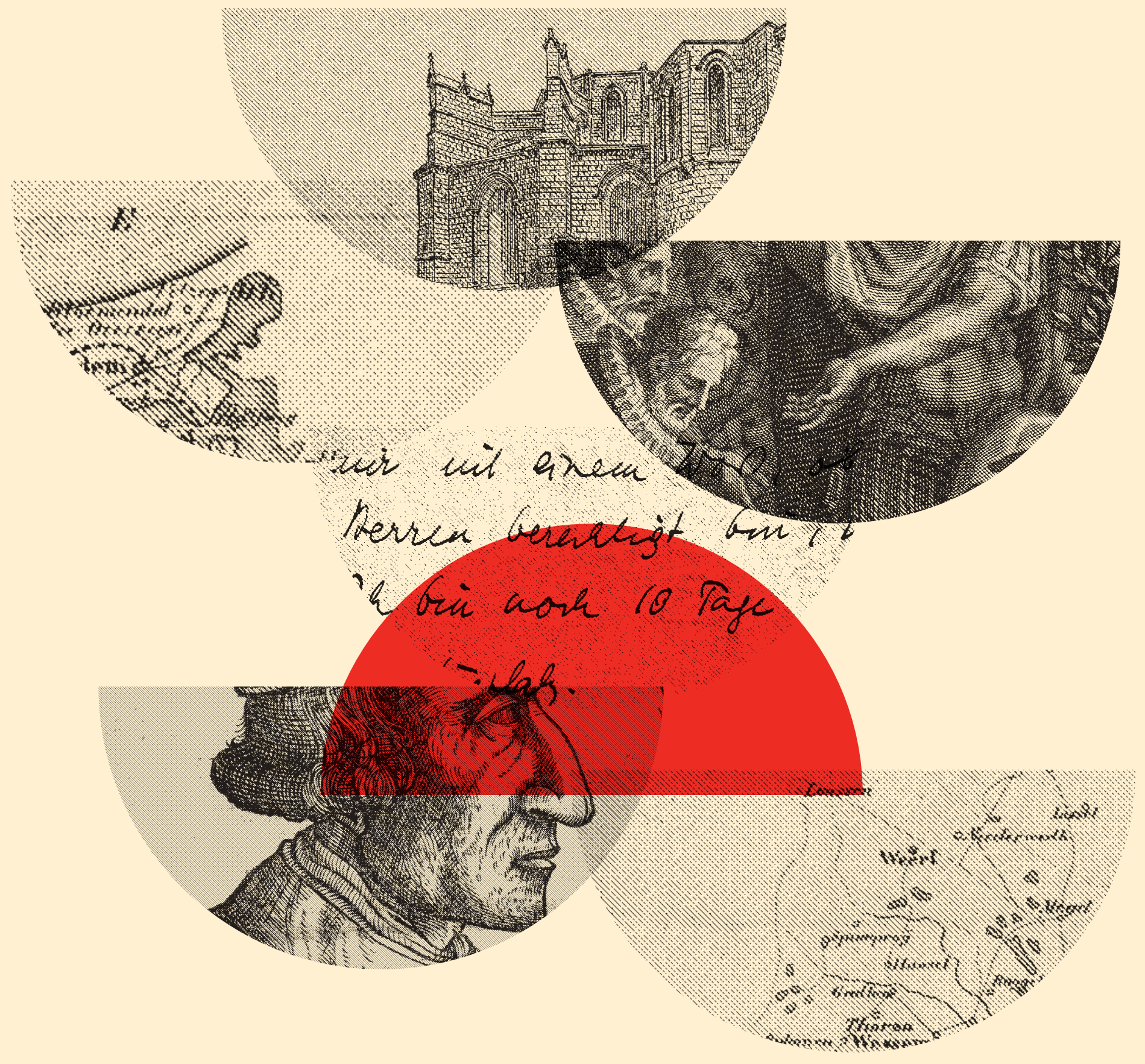




\section{ARRÊTS SUR VING'T-SEP'T IMAGES DE L'EUROPE}

En 1957 étaient signés à Rome les traités portant création de la Communauté économique européenne. Soixante ans après, en 2017, l'Union européenne qui a succédé à la CEE en 1993 (après la signature, un an plus tôt, du traité de Maastricht) est devenue pour beaucoup la matérialisation d'une nouvelle destinée, d'une nouvelle conscience commune, patiemment bâtie depuis la fin de la Seconde Guerre mondiale - pour beaucoup, mais pas pour tous, comme l'a rappelé en 2016 le vote britannique entérinant par referendum la sortie du Royaume-Uni de l'UE et aboutissant à une procédure de retrait qui fut enclenchée en cette même année 2017.

Soixante ans après le traité de Rome, l'avenir de l'Union s'écrit donc à vingt-sept. Derrière ce chiffre, somme toute considérable, apparaît un ensemble démographique, économique et culturel qui ne l'est pas moins, se lit aussi une histoire commune qui s'est constituée par vagues successives et continue d'impacter la manière dont chaque pays, chaque aire linguistique ou culturelle ressent sa propre appartenance à l'Union. Derrière le chiffre des 27 se laisse aussi deviner, aujourd'hui encore et malgré toutes les conséquences de la mondialisation, une mosaïque dont rend bien compte la devise adoptée par l'UE et officiellement proclamée en 2000 par le parlement européen, In varietate concordia ou, pour le dire dans sa traduction française officielle, Unie dans la diversité.

Diversités linguistiques, diversités culturelles, multiplicité des regards : derrière ces termes qui renvoient aux missions mêmes de toute bibliothèque, apparaît en filigrane le désir qu'avait la BNU de commémorer, à sa façon, un des actes fondateurs de la " maison commune européenne ». Dépositaire depuis 2009 du fonds de documentation imprimée du Conseil de l'Europe, animatrice depuis 2013, au niveau du site alsacien, d'un groupe de travail visant à faire de celui-ci un pôle de référence documentaire sur l'Europe, c'est toutefois par le biais de ses collections encyclopédiques qu'elle pouvait, nous semble-t-il, appréhender le mieux cette « union dans la diversité », programmatique aussi d'une ambition intellectuelle. 
L'angle choisi pour construire ce numéro atypique par rapport au format habituel de La Revue a été le suivant : il s'agissait de (re)présenter chaque pays de l'actuelle UE par un document emblématique - de sa construction politique, de sa construction mentale ou culturelle, ou encore de sa contribution à l'idée européenne. Chaque document donne ainsi une image, ou mieux, opère un arrêt sur une image du pays concerné, qui ne saurait le résumer, mais est chaque fois, pensons-nous, suffisamment explicite pour évoquer sa singularité comme sa participation au " concert des nations " européennes. Il va de soi que le hasard (présence ou non d'un document dans les collections de la BNU) et la subjectivité, inévitable, du commentateur ont joué leur rôle... Il n'en demeure pas moins que dans le portrait d'Europe qui s'esquisse à la fin de la lecture, apparaissent des leitmotivs qui pourraient bien avoir sous-tendu la construction de l'Union, voire constituer les bases plus ou moins conscientes qui forgent notre sentiment d'appartenance à cette communauté continentale.

Parmi les fils qui tissent une destinée commune, comment ne pas retenir l'importance du sentiment romantique qui rattacha, tout au long du $19^{\mathrm{e}}$ siècle, l'émancipation des peuples à la redécouverte de la culture populaire où gisait - pour reprendre les mots de l'époque - «l'âme de la nation » ? Herder et Mérimée comptent aujourd'hui encore parmi les passeurs privilégiés de cette quête où la littérature et la musique jouèrent un rôle politique de premier plan. Autre fil persistant, celui de la « république des lettres » née des Lumières européennes, des réflexions de Rousseau sur l'Histoire et de celles de Kant sur la paix perpétuelle, celui aussi des traducteurs qui font circuler les idées et les textes et anticipent les échanges culturels. Enfin, derrière la recherche des racines préludant à l'éveil des nations, pointe aussi en creux le développement des nationalismes, qui devait contribuer naguère, en favorisant l'émergence de nouveaux États, au maintien de l'équilibre en Europe, mais questionne aujourd'hui cette même entité au nom de la sauvegarde de traditions «nationales ». La diversité aurait-elle raison de l'union?

Les vingt-sept arrêts sur image présentés dans ce numéro sont riches, aussi, de cette multiplicité de points de vue. Le choix des documents engage, fait émerger des visions contrastées de l'Europe et de sa construction, et rappelle, au moment où des volontés séparatistes se font jour au sein même des États membres, qu'en politique rien n'est jamais acquis. Mais en même temps, ces vingt-sept images si diverses par leurs époques, leurs supports, leur diffusion ou leurs commanditaires nous montrent aussi l'impossibilité de rompre les liens organiques qu'ont forgés des siècles de construction culturelle commune - la référence antique, les modes de diffusion des savoirs, l'héritage des mouvements intellectuels ou religieux, des conflits et des révolutions.

In varietate concordia : espérons-le pour les décennies, les siècles à venir. En attendant, vingt-sept pays ont accepté de jouer le jeu d'un numéro spécial ; au lecteur de se mettre à son tour à l'épreuve de ces images d'Europe.

\section{Christophe Didier}

